\title{
PReS-FINAL-2283: Systemic lupus erythematosus (SLE) in children and adolescents in pediatric unite, institute of rheumatology Belgrade, Serbia
}

\author{
B Stanimirovic ${ }^{*}$, G Susic ${ }^{2}$, D Novakovic ${ }^{2}$, R Stojanovic ${ }^{2}$ \\ From 20th Pediatric Rheumatology European Society (PReS) Congress \\ Ljubljana, Slovenia. 25-29 September 2013
}

\section{Introduction}

SLE is an autoimmune disease characterized by widespread inflammation of blood vessels and connective tissues.

\section{Objectives}

The aim of this study is to describe the clinical and laboratory manifestations, treatment, complications and disease outcome in children and adolescents with SLE.

\section{Methods}

Medical records of all children and adolescents with SLE treated during 10 years period (between 2001 and 2011) at the Institute of Rheumatology Belgrade were retrospectively reviewed. The collected data included informations about demographic profile, clinical and laboratory manifestations, treatment, complications and disease outcome.

\section{Results}

Thirty seven patients (36 f, $1 \mathrm{~m}$ ) of SLE were reviewed.. The mean age at disease onset was 15 years with a range of 7-19 years. The most common features were mucocutaneus (malar rash in $84,8 \%$, photosensitivity in $69,7 \%$ ); musculoskeletal (arthritis in 87,9\%) and hematological (leucopenia in $75,8 \%$, anemia in $69,7 \%$, Coombs test positive in $18,9 \%$ and, thrombocytopenia in $28 \%$ ). Renal involvement occurred in $45,5 \%$ of children. CNS manifestations in $51,2 \%$. ANA was positive in $97,3 \%$, Anti dsDNA in $81,1 \%$, Anti Sm in $40 \%$ of our patients. Corticosteroid treatment was given in all patients in the form of prednisone $(100 \%)$ and methylprednisolone, iv pulses were applied in $58,8 \%$. Antimalarics were used in $97.3 \%$,

${ }^{1}$ Clinical centar Banjaluka, Banjaluka, Bosnia and Herzegovina

Full list of author information is available at the end of the article azathiophrine in $48,6 \%$, micophenolat-mofetil in $21,6 \%$ and cyclosporine in $3,8 \%$ of children. The most common complications were hypertension, hypercorticism and opportunistic infections. Three patients died during the period of the study, two girls according to antiphospholipid syndrome complications, one of infection (sepsis).

\section{Conclusion}

The most common features were mucocutaneus, musculosceletal and hematological. Less than half of the patients were with renal involvement, although $80 \%$ were anti ds DNA positive. All patients were treated with corticosteroids, and except three with antimalarics. High blood pressure, hypercorticism and opportunistic infections were most common complications. There was no significant difference in clinical and laboratory manifestations, therapy approach and outcome compared to those in most pediatric SLE studies. Low number of patients with renal involvement can be explained by profile of institution.

\section{Disclosure of interest}

None declared.

\section{Authors' details \\ ${ }^{1}$ Clinical centar Banjaluka, Banjaluka, Bosnia and Herzegovina. ${ }^{2}$ Institute of Rheumatology, Belgrade, Serbia.}

Published: 5 December 2013

\section{doi:10.1186/1546-0096-11-S2-P273}

Cite this article as: Stanimirovic et al.: PReS-FINAL-2283: Systemic lupus erythematosus (SLE) in children and adolescents in pediatric unite, institute of rheumatology Belgrade, Serbia. Pediatric Rheumatology 2013 11(Suppl 2):P273. 